\title{
Influence of thyroid dysfunction on brain natriuretic peptide level in health examination participants
}

\author{
Kenji Ohba ${ }^{1), 2)}$, Eisaku Okada ${ }^{3)}$, Yoshie Goto ${ }^{1)}$, Sadako Suzuki ${ }^{1)}$, Masashi Machii ${ }^{1)}$, Daishi Nonaka ${ }^{1)}$, \\ Akio Matsushita' ${ }^{2)}$, Shigekazu Sasaki²), Takafumi Suda ${ }^{2)}$, Yutaka Oki ${ }^{4)}$ and Hiroyuki Takase ${ }^{1)}$ \\ 1) Department of Internal Medicine, Enshu Hospital, 1-1-1 Chuo, Naka-ku, Hamamatsu, Shizuoka 430-0929, Japan \\ 2) Second Division, Department of Internal Medicine, Hamamatsu University School of Medicine, 1-20-1 Handayama, Higashi-ku, \\ Hamamatsu, Shizuoka 431-3192, Japan \\ 3) Department of Community Health and Preventive Medicine, Hamamatsu University School of Medicine, 1-20-1 Handayama, \\ Higashi-ku, Hamamatsu, Shizuoka 431-3192, Japan \\ 4) Department of Family and Community Medicine, Hamamatsu University School of Medicine, 1-20-1 Handayama, Higashi-ku, \\ Hamamatsu, Shizuoka 431-3192, Japan
}

\begin{abstract}
The tissue-specific circulating markers of thyroid hormone action on cardiac function have not been established. Although the relationship between thyroid function and plasma brain natriuretic peptide (BNP) levels has been evaluated in patients with thyroid disorders, the relationship between these parameters in the general population has not been yet studied. We conducted retrospective cohort study by health examination with concurrent measurements of TSH, free T4, body mass index, systolic blood pressure, hemoglobin, and estimated glomerular filtration rate from participants who visited the Department of Health Checkup, Enshu Hospital between July 2008 and March 2017. After participants with abnormal electrocardiogram and/or any history of cardiac disease were excluded, 2,807 individuals were subjected. Multivariate analyses demonstrated that, when compared to euthyroidism $(n=2,629)$, the increase in BNP levels was significant in overt thyrotoxicosis $(n=21)$ but not in subclinical thyrotoxicosis $(n=53)$ or subclinical hypothyroidism $(n=97)$. Interestingly, the standardized partial regression coefficient was the smallest for thyroid function category (overt thyrotoxicosis compared to euthyroidisim; $\beta=0.048, p=0.006$ ) among the independent variables including age, body mass index, systolic blood pressure, and hemoglobin. In longitudinal comparison, we identified 986 participants who had sequential data on the measurements and were stable as euthyroidism and subclinical hypothyroidism. Their annual percent change in BNP demonstrated no significant differences. In conclusion, a direct stimulatory effect of thyroid hormone on the secretion (or production) of BNP was confirmed even in a large number of health examination participants.
\end{abstract}

Key words: Brain natriuretic peptide, Heart, Thyrotoxicosis, Biomarker, Retrospective cohort study

PREVIOUS STUDIES have suggested that circulating levels of thyroid hormone (TH) and TSH may not always reflect TH action in specific peripheral tissues [1], and many cell culture and/or in vivo animal model studies have reported that the effects of $\mathrm{TH}$ are determined not only by serum $\mathrm{TH}$ concentrations but also by expression levels of several key regulators, such as TH transporters and iodothyronine deiodinases $[2,3]$. Although the myocardium is a major target for THs [4], the tissue-specific circulating markers of $\mathrm{TH}$ action have not been identified [5].

Submitted Aug. 28, 2019; Accepted Dec. 13, 2019 as EJ19-0380 Released online in J-STAGE as advance publication Jan. 18, 2020 Correspondence to: Hiroyuki Takase, MD, PhD, Department of Internal Medicine, Enshu Hospital, 1-1-1 Chuo, Naka-ku, Hamamatsu, Shizuoka 430-0929, Japan.

E-mail: h-takase.ken@shizuokakouseiren.jp
The brain natriuretic peptide (BNP) is critical for ventricular cardiomyocytes in response to volume expansion, pressure overload, and elevated end-diastolic pressure. BNP is synthesized as a precursor protein (pro$\mathrm{BNP}$ ) and is cleaved into the physiologically active BNP and a biologically inactive N-terminal fragment (NT-proBNP). Circulating levels of these peptides are strongly associated with both severity and prognosis in heart failure [6-8]. Apart from cardiovascular disorders, BNP levels are also known to be affected by aging, gender, obesity, anemia, and kidney function [9].

Several studies have reported that circulating BNP and/or NT-proBNP levels are elevated in patients with thyrotoxicosis [10-18], and the major underlying mechanism is recognized to be the induction of hemodynamic change, such as during heart failure, due to elevated TH levels $[12,17]$. In addition to these indirect effects on 
plasma BNP, a direct stimulatory effect of TH on ventricular cardiomyocytes has also been reported [10, 12, 14, 16-19]. Although the relationship between thyroid function and BNP has been evaluated in patients with thyroid disorders, the significance of these parameters in the general population has not been yet studied. Several studies have demonstrated that the effect of TH changes between acute and chronic hyperthyroidism [1, 20, 21]; however, the long-term direct effect of $\mathrm{TH}$ on the secretion of BNP is also poorly kown.

Thus, to investigate the independent influence of $\mathrm{TH}$ status on plasma BNP levels, we conducted a retrospective cohort study in a large sample of medical checkup participants.

\section{Materials and Methods}

We retrospectively reviewed concurrent measurements of TSH, free T4 (FT4), BNP, body mass index (BMI), systolic blood pressure (SBP), hemoglobin, and estimated glomerular filtration rate (eGFR) from participants who visited the Department of Health Checkup, Enshu Hospital, between July 2008 and March 2017. Their medical records were manually reviewed. To evaluate the long-term direct effect of $\mathrm{TH}$ on the secretion of BNP, a longitudinal comparison was further performed using data from participants for whom periodic and sequential values for the parameters mentioned above were available. Medical histories were reported by the subjects themselves on questionnaires. As plasma BNP concentrations are critically affected by cardiac dysfunction [6], exclusion criteria were as follows: abnormal electrocardiogram and/or any history of cardiac diseases, such as coronary artery disease, arrhythmia, and congestive heart failure. Participants with thyroid dysfunction were included, irrespective of whether they were receiving drug therapy, including antithyroid drugs.

Blood samples were collected in the early morning between 8:30 and 9:30 AM after fasting for at least 12 hours. Plasma BNP concentration was determined using a commercially available chemiluminescence enzyme immunoassay (MI02 Shionogi BNP kit; Shionogi, Osaka, Japan). Serum TSH and FT4 concentrations were measured using an electrochemiluminescent immunoassay kit (ECLusys TSH or ECLusys FT4, Roche Diagnostics, Tokyo, Japan). Reference ranges used were as follows: BNP $<18.4 \mathrm{pg} / \mathrm{mL}$, TSH $0.5-5.0 \mu \mathrm{IU} / \mathrm{mL}$, and FT4 0.9-1.7 ng/dL. Thyrotoxicosis was defined as TSH $<0.5 \mu \mathrm{IU} / \mathrm{mL}$ and was classified as either overt (OT) with high FT4 or subclinical (SCT) with FT4 within the reference range. Hypothyroidism was defined as TSH $>5.0 \mu \mathrm{IU} / \mathrm{mL}$ and was categorized as either overt $(\mathrm{OH})$ with low FT4 or subclinical (SCH) with FT4 within the reference range.
Data are expressed as mean \pm standard deviation or medians with interquartile range in parentheses. BNP, TSH, and FT4 levels were Box-Cox transformed before statistical analyses because of their skewed distributions. One-way analysis of variance followed by Dunnett's post hoc test, chi-square test, unpaired $t$-test, Fisher's exact test, or Mann-Whitney test was used for comparisons among the multiple thyroid dysfunction categories. The relationship between BNP (Box-Cox transformed) and other variables such as age, gender, BMI, SBP, hemoglobin, and eGFR, was examined using Pearson's correlation test and/or multivariable regression analysis. All statistical analyses were conducted using the JMP (version 11.0, SAS Institute, Inc., Cary, NC), the GraphPad PRISM (version 6.0, GraphPad Software, Inc., San Diego, CA) or the BellCurve for Excel (version 1.01, Social Survey Research Information Co., Ltd., Tokyo, Japan) software. By entering effect size $=0.35$, $\alpha=0.05, \beta=0.2$, and number of groups $=5$ (OT, SCT, euthyroidism (EU), $\mathrm{SCH}$, and $\mathrm{OH})$, the minimum number of participants in each group for one-way analysis of variance was calculated as 20.5 using $\mathrm{R}$ software [22].

The study protocol was approved by the Ethics Committee of Enshu Hospital, and was congruent with the Declaration of Helsinki. Written informed consent was obtained from every participant.

\section{Results}

We selected 2,807 individuals, and their baseline characteristics are summarized in Table 1. Participants with $\mathrm{OH}(n=7)$ were excluded because of the small group size. Among the multiple thyroid dysfunction categories including OT $(n=21)$, SCT $(n=53)$, EU $(n=2,629)$, and $\mathrm{SCH}(n=97)$, significant differences were observed in plasma BNP concentrations and in several factors known to affect plasma BNP such as age, hemoglobin, and eGFR (Table 1). Post hoc analysis revealed that BNP levels were significantly higher in OT and $\mathrm{SCH}$ groups than in the EU group. Subsequent multivariate analyses adjusted by age, gender, and variables with a $p$-value $<0.05$ after univariate analysis (hemoglobin and eGFR in Table 1) demonstrated that, when compared to EU, an increase in BNP levels was significant in OT but not in $\mathrm{SCH}$ group (Table 2). In patients with OT, there was a positive association between circulating levels of BNP and FT4 after Box-Cox transformation $\left(r^{2}=0.302, p<\right.$ 0.001 ; Fig. 1). It must be noted here that the standardized partial regression coefficient $(\beta)$ was the smallest for thyroid function category (OT compared to $\mathrm{EU} ; \beta=0.048, p$ $=0.006$ ) among the independent variables tested, including age, BMI, SBP, and hemoglobin (Table 3).

In the longitudinal comparison, 1,082 of the 2,807 par- 
Table 1 Baseline characteristics of the study population $(n=2,800)$

\begin{tabular}{|c|c|c|c|c|c|}
\hline Variables at baseline & OT & $\mathrm{SCT}$ & EU & $\mathrm{SCH}$ & $p$ values \\
\hline Participants & 21 & 53 & 2,629 & 97 & \\
\hline Age (years) $)^{\text {a) }}$ & $51.6 \pm 13.5$ & $52.8 \pm 11.6$ & $55.0 \pm 11.4$ & $61.9 \pm 11.8^{* * *}$ & $<0.001$ \\
\hline Gender (male/female) $)^{\text {b) }}$ & $10 / 11$ & $5 / 48$ & $769 / 1,860$ & $26 / 71$ & 0.463 \\
\hline $\left.\mathrm{TSH}(\mathrm{mIU} / \mathrm{L})^{\mathrm{c}}\right)$ & $0.0(0.0-0.1)^{* * *}$ & $0.3(0.1-0.4)^{* * *}$ & $1.7(1.2-2.4)$ & $6.0(5.3-7.3)^{* * *}$ & $<0.001$ \\
\hline FT4 (ng/dL) $)^{c}$ & $2.1(1.9-2.8)^{* * *}$ & $1.3(1.2-1.5)^{* * *}$ & $1.2(1.2-1.3)$ & $1.1(1.1-1.3)^{* * *}$ & $<0.001$ \\
\hline $\left.\mathrm{BNP}(\mathrm{pg} / \mathrm{mL})^{\mathrm{c}}\right)$ & $17.2(10.7-23.5)^{*}$ & $13.4(7.0-23.3)$ & $11.9(7.0-19.0)$ & $15.8(9.3-30.1)^{* * *}$ & $<0.001$ \\
\hline Body mass index $\left(\mathrm{kg} / \mathrm{m}^{2}\right)^{\mathrm{a})}$ & $21.9 \pm 3.4$ & $22.4 \pm 3.1$ & $22.5 \pm 3.4$ & $22.3 \pm 3.0$ & 0.832 \\
\hline Systolic blood pressure $(\mathrm{mmHg})^{\mathrm{a})}$ & $122.4 \pm 15.3$ & $121.2 \pm 14.0$ & $122.1 \pm 15.8$ & $125.0 \pm 15.2$ & 0.347 \\
\hline Hemoglobin $(\mathrm{g} / \mathrm{dL})^{\mathrm{a})}$ & $13.1 \pm 1.2$ & $13.4 \pm 1.4$ & $13.4 \pm 1.3$ & $13.0 \pm 1.4^{*}$ & 0.027 \\
\hline $\mathrm{eGFR}\left(\mathrm{mL} / \mathrm{min} / 1.73 \mathrm{~m}^{2}\right)^{\mathrm{a})}$ & $95.1 \pm 39.3^{* * *}$ & $83.4 \pm 13.2^{*}$ & $77.9 \pm 13.7$ & $71.0 \pm 14.5^{* * *}$ & $<0.001$ \\
\hline
\end{tabular}

The continuous variables are expressed as a) mean \pm standard deviation or ${ }^{\mathrm{c})}$ medians with interquartile range in brackets. The $p$-values were derived using a) one-way analysis of variance, ${ }^{\text {b) }}$ chi-square test, or ${ }^{c}$ one-way analysis of variance after Box-Cox transformation. ${ }^{*}, p<0.05$; ***, $p<0.001$, compared with euthyroidism (EU) by Dunnett's multiple comparison test.

Abbreviations: OT, overt thyrotoxicosis; SCT, subclinical thyrotoxicosis; SCH, subclinical hypothyroidism; FT4, free thyroxine; BNP, brain natriuretic peptide; eGFR, estimated glomerular filtration rate.

Table 2 Differences in brain natriuretic peptide (BNP) levels at baseline in the groups, compared to the euthyroid group

\begin{tabular}{lcccc}
\hline & OT & SCT & EU & SCH \\
\hline Participants & 21 & 53 & 2,629 & 97 \\
Crude Analysis & $0.40^{*}[0.09$ to 0.71$]$ & $0.09^{*}[0.09$ to 0.71$]$ & Ref. & $0.30^{* *}[0.15$ to 0.44$]$ \\
Adjusted analysis $^{1)}$ & $0.45^{* *}[0.16$ to 0.74$]$ & $0.10[-0.08$ to 0.28$]$ & Ref. & $0.14[-0.00$ to 0.27$]$ \\
Adjusted analysis $^{2)}$ & $0.41^{* *}[0.13$ to 0.69$]$ & $0.08[-0.09$ to 0.26$]$ & Ref. & $0.08[-0.05$ to 0.21$]$ \\
\hline
\end{tabular}

After Box-Cox transformed BNP was inserted into multiple linear regression analyses, partial regression coefficient $(B)$ for each thyroid dysfunction category, compared to the euthyroid (EU) group, was obtained. Data in square brackets are $95 \%$ confidence intervals. ${ }^{1)}$ Adjusted analysis: age and gender. ${ }^{2}$ Adjusted analysis: age, gender, hemoglobin, and estimated glomerular filtration rate.

Abbreviations: OT, overt thyrotoxicosis; SCT, subclinical thyrotoxicosis; $\mathrm{SCH}$, subclinical hypothyroidism; Ref., reference.

*, $p<0.05 ; * *, p<0.01$.

ticipants were followed over a period of $22.3 \pm 16.5$ months. Further, we identified 994 participants whose thyroid function results were available at both baseline and follow-up, and remained stable as OT to OT (OTOT; $n=2$ ), SCT to SCT (SCT-SCT; $n=4$ ), EU to EU (EU-EU; $n=963$ ), $\mathrm{SCH}$ to $\mathrm{SCH}$ ( $\mathrm{SCH}-\mathrm{SCH} ; n=23$ ), and $\mathrm{OH}$ to $\mathrm{OH}(\mathrm{OH}-\mathrm{OH} ; n=2)$. Participants with OTOT, SCT-SCT, or OH-OH were excluded because of the small group size. Thus, the final sample size for the longitudinal comparison was 986 . Table 4 summarizes baseline and follow-up characteristics of participants with EU-EU and SCH-SCH. Their annual percent change in BNP was not significantly different $(p=0.128)$. Multivariate analyses demonstrated that hemoglobin and eGFR values at baseline were significantly associated with changes in BNP levels during follow-up (Table 5).

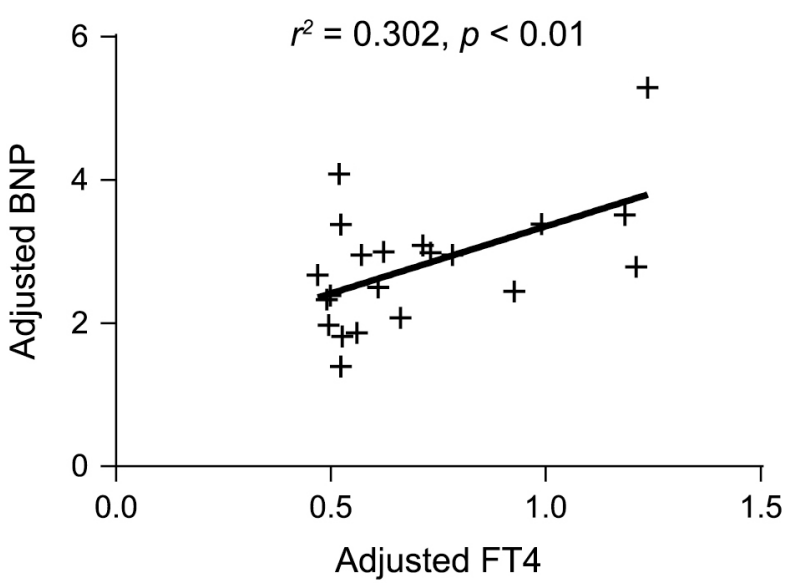

Fig. 1 Scattergram showing the relationship between circulating levels of brain natriuretic peptide (BNP) and free thyroxine (FT4) after Box-Cox transformation in participants with overt thyrotoxicosis. The $r^{2}$ - and $p$ values were derived using Pearson's correlation analysis. The solid line is the regression line. 
Table 3 Predictors of elevated brain natriuretic peptide (BNP) levels in participants with overt thyrotoxicosis and euthyroidism

\begin{tabular}{lrc}
\hline Variables & $\beta$ & $p$ \\
\hline Age & $0.303<0.001$ \\
Gender (male/female) & -0.034 & 0.113 \\
Thyroid function category (OT compared to EU) & 0.048 & 0.006 \\
Body mass index $\left(\mathrm{kg} / \mathrm{m}^{2}\right)$ & $-0.139<0.001$ \\
Systolic blood pressure $(\mathrm{mmHg})$ & $0.105<0.001$ \\
Hemoglobin $(\mathrm{g} / \mathrm{dL})$ & $-0.277<0.001$ \\
eGFR $\left(\mathrm{mL} / \mathrm{min} / 1.73 \mathrm{~m}^{2}\right)$ & -0.005 & 0.791 \\
\hline
\end{tabular}

The relationship between Box-Cox transformed BNP and predictor variables was examined using multivariable regression analysis $\left(R^{2}=0.218, F=104.7, p<0.001\right)$.

Abbreviations: OT, overt thyrotoxicosis; EU, euthyroidism; eGFR, estimated glomerular filtration rate.

\section{Discussion}

In this study, we report on two important results obtained by concurrently analyzing $\mathrm{TH}$ status, plasma BNP, and known confounders in participants with no clinical evidence of cardiac dysfunction. First, we demonstrate that, compared to the EU group, a significant elevation in plasma BNP was identified in the OT group even after adjustment (Table 2). Evidence for a direct stimulatory effect of THs on the secretion of BNP is available; specifically, Kohno et al. were the first to demonstrate that THs released BNP into the medium in a primary culture of newborn rat ventricular cardiomyocytes [10]. Additionally, an in vitro study by Liang et al. identified the BNP gene as a transcriptional target of THs [19]. The direct effect was further investigated in

Table 4 Characteristics at baseline and follow-up in the longitudinal comparison $(n=986)$

\begin{tabular}{|c|c|c|c|}
\hline Variables & EU-EU & $\mathrm{SCH}-\mathrm{SCH}$ & $p$ values \\
\hline Participants & 963 & 23 & \\
\hline Duration of follow-up (months) ${ }^{\mathrm{a})}$ & $21.9 \pm 16.2$ & $23.1 \pm 14.5$ & 0.728 \\
\hline \multicolumn{4}{|l|}{ At baseline } \\
\hline Age $(\text { years })^{a)}$ & $56.2 \pm 9.7$ & $63.0 \pm 9.3$ & $<0.001$ \\
\hline Gender (male/female) $\left.{ }^{b}\right)$ & $228 / 735$ & $7 / 16$ & 0.460 \\
\hline $\mathrm{TSH}(\mathrm{mIU} / \mathrm{L})^{\mathrm{c})}$ & $1.7(1.3-2.4)$ & $7.1(6.2-9.1)$ & $<0.001$ \\
\hline FT4 $\left.(n g / d L)^{c}\right)$ & $1.2(1.2-1.3)$ & $1.1(1.0-1.3)$ & 0.002 \\
\hline $\mathrm{BNP}(\mathrm{pg} / \mathrm{mL})^{\mathrm{c})}$ & $12.5(7.8-19.0)$ & $11.7(7.6-30.2)$ & 0.591 \\
\hline Body mass index $\left(\mathrm{kg} / \mathrm{m}^{2}\right)^{\mathrm{a})}$ & $22.5 \pm 3.2$ & $22.8 \pm 2.7$ & 0.606 \\
\hline Systolic blood pressure $(\mathrm{mmHg})^{\mathrm{a})}$ & $122.7 \pm 15.4$ & $125.7 \pm 15.5$ & 0.362 \\
\hline Hemoglobin $(\mathrm{g} / \mathrm{dL})^{\mathrm{a})}$ & $13.3 \pm 1.2$ & $13.3 \pm 1.4$ & 0.904 \\
\hline eGFR $\left(\mathrm{mL} / \mathrm{min} / 1.73 \mathrm{~m}^{2}\right)^{\mathrm{a})}$ & $78.3 \pm 13.8$ & $64.5 \pm 13.6$ & $<0.001$ \\
\hline \multicolumn{4}{|l|}{ At follow-up } \\
\hline Age (years) ${ }^{a)}$ & $58.0 \pm 9.6$ & $65.1 \pm 9.6$ & $<0.001$ \\
\hline $\mathrm{TSH}(\mathrm{mIU} / \mathrm{L})^{\mathrm{c})}$ & $1.7(1.3-2.5)$ & $6.5(5.9-7.5)$ & $<0.001$ \\
\hline FT4 $\left.(n g / d L)^{c}\right)$ & $1.2(1.2-1.3)$ & $1.2(1.1-1.3)$ & 0.004 \\
\hline $\mathrm{BNP}(\mathrm{pg} / \mathrm{mL})^{\mathrm{c})}$ & $11.7(7.1-19.1)$ & $14.2(8.4-26.6)$ & 0.244 \\
\hline Body mass index $\left(\mathrm{kg} / \mathrm{m}^{2}\right)^{\mathrm{a})}$ & $22.5 \pm 3.4$ & $23.1 \pm 3.0$ & 0.364 \\
\hline Systolic blood pressure $(\mathrm{mmHg})^{\mathrm{a})}$ & $121.4 \pm 14.4$ & $124.8 \pm 11.1$ & 0.260 \\
\hline Hemoglobin $(\mathrm{g} / \mathrm{dL})^{\mathrm{a})}$ & $13.4 \pm 1.2$ & $13.6 \pm 1.2$ & 0.420 \\
\hline eGFR $\left(\mathrm{mL} / \mathrm{min} / 1.73 \mathrm{~m}^{2}\right)^{\mathrm{a})}$ & $76.8 \pm 13.3$ & $63.5 \pm 15.0$ & $<0.001$ \\
\hline Annual percent change in BNP $(\%)^{a)}$ & $-0.5 \pm 21.7$ & $6.5 \pm 19.6$ & 0.128 \\
\hline
\end{tabular}

Participants who continued to remain in their initial thyroid dysfunction categories during follow-up, i.e., from subclinical hypothyroidism $(\mathrm{SCH})$ to $\mathrm{SCH}$ were designated as $\mathrm{SCH}-\mathrm{SCH}$, and euthyroidism (EU) to EU as EU-EU.

The continuous variables were expressed as a)mean \pm standard deviation or c)medians with interquartile range in brackets. The $p$-values derived from ${ }^{\text {a) }}$ unpaired $t$-test, ${ }^{\mathrm{b}}$ Fisher's exact test, or ${ }^{c}$ Mann-Whitney test between EU-EU and SCH-SCH groups. Annual percent change in serum brain natriuretic peptide (BNP) levels was calculated as follows: \{(Box-Cox transformed BNP at follow-up/ Box-Cox transformed BNP at baseline) -1$\} \times 12$ /length of follow-up months.

Abbreviations: FT4, free thyroxine; eGFR, estimated glomerular filtration rate. 
clinical studies on participants with thyroid disorders but with no clinical or echocardiographic evidence of ventricular dysfunction, and these studies showed contrasting results. Two studies have identified a significant increase in circulating BNP or NT-proBNP levels in patients with OT $[14,16]$. Pakula et al. have further confirmed this significant elevation in BNP in patients with SCT [18]. However, Wei et al. have reported no significant elevation in BNP, even in patients with OT, compared to control subjects [12]. These inconsistent results can be explained by methodological differences. For example, confounders of circulating BNP or NT-proBNP were rarely taken into consideration in some previous studies, and a small number of control subjects was enrolled to compare the circulating levels of these hormones with those observed in patients with thyroid disorders.

We have evaluated the relationship between TH status and plasma BNP levels in a large sample of the general population and shown a direct stimulatory effect of THs on BNP under conditions of OT (Table 2). To the best of our knowledge, this is the first study of its kind. The strength of our study lies in its strict inclusion and exclusion criteria, which helped exclude any possible interference from known contributing factors that affect plasma BNP, including cardiovascular disorders such as heart failure and atrial fibrillation. Additionally, the $\beta$ value for OT was smallest among several independent factors

Table 5 Predictors of increase in annual rate of change in brain natriuretic peptide (BNP) levels in participants with subclinical thyroid disorders and euthyroidism

\begin{tabular}{|c|c|c|}
\hline Variables & $\beta$ & $p$ \\
\hline Duration of follow-up (months) & 0.055 & 0.087 \\
\hline Age at baseline & -0.036 & 0.320 \\
\hline Gender (male/female) & -0.039 & 0.304 \\
\hline $\begin{array}{l}\text { Thyroid function category (SCH-SCH compared } \\
\text { to EU-EU) }\end{array}$ & 0.040 & 0.212 \\
\hline Body mass index at baseline $\left(\mathrm{kg} / \mathrm{m}^{2}\right)$ & 0.013 & 0.690 \\
\hline Systolic blood pressure at baseline $(\mathrm{mmHg})$ & -0.021 & 0.561 \\
\hline Hemoglobin at baseline $(\mathrm{g} / \mathrm{dL})$ & 0.144 & $<0.001$ \\
\hline eGFR at baseline $\left(\mathrm{mL} / \mathrm{min} / 1.73 \mathrm{~m}^{2}\right)$ & -0.084 & 0.011 \\
\hline
\end{tabular}

The relationship between predictors and annual rate of change in BNP levels, calculated as (Box-Cox transformed BNP at followup/ Box-Cox transformed BNP at baseline) -1$\} \times 12$ /length of follow-up months, was examined using multivariable regression analysis $\left(R^{2}=0.029, F=3.647, p<0.001\right)$. Participants who continued to remain in their initial thyroid dysfunction categories during follow-up, i.e., from subclinical hypothyroidism ( $\mathrm{SCH}$ ) to $\mathrm{SCH}$ were designated as $\mathrm{SCH}-\mathrm{SCH}$, and euthyroidism (EU) to $\mathrm{EU}$ as EU-EU.

Abbreviations: eGFR, estimated glomerular filtration rate. associated with elevated BNP (i.e., age, hemoglobin, BMI, and SBP), suggesting that plasma BNP concentrations may not be a sensitive marker of $\mathrm{TH}$ action on the myocardium. In contrast, age was the strongest contributing factor in our study. A significantly elevated BNP in patients with $\mathrm{SCH}$ before adjustment (Table 2) could be explained by their older ages (Table 1). Together, these results suggest that factors other than the $\mathrm{TH}$ greatly contribute to BNP levels under several conditions, and that not only cardiovascular dysfunction but also other contributing factors should be taken into consideration when BNP levels are assessed in patients with thyroid disorders.

Second, we evaluated whether sustained thyroid dysfunction is associated with plasma BNP levels. To the best of knowledge, this is also a first-of-its-kind study. Importantly, the annual percent change in BNP was not significantly different between $\mathrm{SCH}-\mathrm{SCH}$ and EU-EU groups (Table 4). Several serum markers have been used to assess the effect of $\mathrm{TH}$ on peripheral tissues in patients with thyroid disorders. However, in contrast to serum TSH concentration, accurate tests to specifically assess the impact of $\mathrm{TH}$ on peripheral tissues are not available due to their lack of sufficient specificity or sensitivity. Possible explanation(s) for this include alterations in metabolic changes produced by factors not related to $\mathrm{TH}$; for example, genetic and dietary factors affect serum cholesterol and triglyceride concentrations. It has also been reported that circulating levels of alkaline phosphatase (ALP) and urinary creatinine ( $\mathrm{Cr}$ ) are not sensitive to thyroid dysfunctions because they are altered only in response to substantial changes in TH concentration [5]. Similar to ALP and urinary Cr, our study demonstrates that overt thyroid dysfunction is required for increasing plasma BNP levels. Further studies focused on identification and characterization of cardiomyocyte-specific circulating markers of $\mathrm{TH}$ action may improve diagnosis and treatment in patients with thyroid disorders.

This study has the following limitations. First, echocardiography was not performed to assess cardiovascular status. We excluded hemodynamic dysfunction by electrocardiography in addition to medical history. It was reported that left ventricular dysfunction is unlikely to be present if the electrocardiogram is normal [23]. Second, as some of the participants undergoing medical treatments for non-cardiac disorders were included in this study, the influence of medications cannot be excluded.

In conclusion, a direct stimulatory effect of overt thyrotoxicosis on the secretion (or production) of BNP was confirmed even in a large number of health examination participants. Subclinical hypothyroidisim is not associated with a change in BNP during follow-up. Further studies focused on identification and characterization of 
cardiomyocyte-specific circulating markers of $\mathrm{TH}$ action may improve diagnosis and treatment in patients with thyroid disorders.

\section{Disclosure}

None of the authors have any potential conflicts of interest associated with this research.

\section{References}

1. Ohba K, Sinha RA, Singh BK, Iannucci LF, Zhou J, et al. (2017) Changes in hepatic TRbeta protein expression, lipogenic gene expression, and long-chain acylcarnitine levels during chronic hyperthyroidism and triiodothyronine withdrawal in a mouse model. Thyroid 27: 852-860.

2. Cheng SY, Leonard JL, Davis PJ (2010) Molecular aspects of thyroid hormone actions. Endocr Rev 31: 139170.

3. Brent GA (2012) Mechanisms of thyroid hormone action. J Clin Invest 122: 3035-3043.

4. Kahaly GJ, Dillmann WH (2005) Thyroid hormone action in the heart. Endocr Rev 26: 704-728.

5. Refetoff S, Weiss RE, Usala SJ (1993) The syndromes of resistance to thyroid hormone. Endocr Rev 14: 348-399.

6. Mukoyama M, Nakao K, Hosoda K, Suga S, Saito Y, et al. (1991) Brain natriuretic peptide as a novel cardiac hormone in humans. Evidence for an exquisite dual natriuretic peptide system, atrial natriuretic peptide and brain natriuretic peptide. J Clin Invest 87: 1402-1412.

7. Takase H, Toriyama T, Sugiura T, Takahashi A, Sugiyama M, et al. (2007) Brain natriuretic peptide detects cardiac abnormalities in mass screening. Eur J Clin Invest 37: 257-262.

8. Nakagawa Y, Nishikimi T, Kuwahara K (2019) Atrial and brain natriuretic peptides: hormones secreted from the heart. Peptides 111: 18-25.

9. Takase H, Dohi Y (2014) Kidney function crucially affects B-type natriuretic peptide (BNP), N-terminal proBNP and their relationship. Eur J Clin Invest 44: 303-308.

10. Kohno M, Horio T, Yasunari K, Yokokawa K, Ikeda M, et al. (1993) Stimulation of brain natriuretic peptide release from the heart by thyroid hormone. Metabolism 42: 1059 1064.

11. Schultz M, Faber J, Kistorp C, Jarlov A, Pedersen F, et al. (2004) N-terminal-pro-B-type natriuretic peptide (NT-pro$\mathrm{BNP})$ in different thyroid function states. Clin Endocrinol (Oxf) 60: 54-59.

12. Wei T, Zeng C, Tian Y, Chen Q, Wang L (2005) B-type natriuretic peptide in patients with clinical hyperthyroidism. $J$ Endocrinol Invest 28: 8-11.

13. Ozmen B, Ozmen D, Parildar Z, Mutaf I, Bayindir O (2007) Serum N-terminal-pro-B-type natriuretic peptide (NT-pro-BNP) levels in hyperthyroidism and hypothyroid- ism. Endocr Res 32: 1-8.

14. Arikan S, Tuzcu A, Gokalp D, Bahceci M, Danis R (2007) Hyperthyroidism may affect serum N-terminal pro-B-type natriuretic peptide levels independently of cardiac dysfunction. Clin Endocrinol (Oxf) 67: 202-207.

15. Mayer O Jr., Simon J, Cech J, Rosolova H, Hrbkova J, et al. (2008) Even mild changes in free thyroxine could influence the degree of heart failure measured by its biological surrogates. Physiol Res 57: 525-529.

16. Ertugrul DT, Gursoy A, Sahin M, Unal AD, Pamuk B, et al. (2008) Evaluation of brain natriuretic peptide levels in hyperthyroidism and hypothyroidism. J Natl Med Assoc 100: 401-405.

17. Kato K, Murakami H, Isozaki O, Tsushima T, Takano K (2009) Serum concentrations of BNP and ANP in patients with thyrotoxicosis. Endocr J 56: 17-27.

18. Pakula D, Marek B, Kajdaniuk D, Krysiak R, Kos-Kudla B, et al. (2011) Plasma levels of NT-pro-brain natriuretic peptide in patients with overt and subclinical hyperthyroidism and hypothyroidism. Endokrynol Pol 62: 523-528.

19. Liang F, Webb P, Marimuthu A, Zhang S, Gardner DG (2003) Triiodothyronine increases brain natriuretic peptide (BNP) gene transcription and amplifies endothelindependent BNP gene transcription and hypertrophy in neonatal rat ventricular myocytes. J Biol Chem 278: 15073-15083.

20. Piatnek-Leunissen DA, Leunissen RL (1969) Liver mitochondrial function in acute vs. chronic hyperthyroidism. Endocrinology 84: 456-461.

21. Ohba K, Leow MK, Singh BK, Sinha RA, Lesmana R, et al. (2016) Desensitization and incomplete recovery of hepatic target genes after chronic thyroid hormone treatment and withdrawal in male adult mice. Endocrinology 157: $1660-1672$.

22. R Core Team (2019) R: A language and environment for statistical computing. R Foundation for Statistical Computing, Vienna, Austria. Available from: https://cran.rproject.org Cited 15 October 2019.

23. Davie AP, Francis CM, Love MP, Caruana L, Starkey IR, et al. (1996) Value of the electrocardiogram in identifying heart failure due to left ventricular systolic dysfunction. BMJ 312: 222. 